\title{
SEXO, GÉNERO Y BIOLOGÍA
}

\author{
JOAQUÍN DE JUAN HERRERO Y ROSA Ma PÉREZ CAÑAVERAS
}

Universidad de Alicante

«El sexo anatómico, la identidad, la preferencia sexual y el comportamiento masculino y femenino, son todas cualidades independientes que se combinan de formas diversas para distin-

tas personas».

Rachel Pollack ${ }^{1}$

\section{INTRODUCCIÓN}

Hemos escrito este artículo con tres propósitos fundamentales: (1) romper la dicotomía tradicional entre el discurso "científico» y el "humanístico», (2) centrar el estudio desde la concepción de que los humanos formamos un continuum con el resto de los organismos del planeta y (3) manifestar un respeto absoluto a las creencias de cada uno, utilizando los datos aquí aportados como fuente para la reflexión y el debate pero nunca con el objetivo de convencer, dogmatizar y menos discriminar. Con esos planteamientos hemos dividido el trabajo en los siguientes apartados:

- Nosotros los organismos: una aproximación a la biología

- Concepto de sexo y de sexualidad

- Identidad de género y papel de género

- Orientación sexual

- Sexo, género y neurociencias

\section{NOSOTROS LOS ORGANISMOS: APROXIMACIÓN A LA BIOLOGÍA}

En general, cuando abrimos un libro de biología o le pedimos a un biólogo que nos defina su disciplina, lo habitual es que nos encontremos con frases como ésta, la Biología es la ciencia de la vida. Lo malo es que esta definición, automáticamente, nos lleva a otra pregunta todavía más difícil de contestar: ¿qué es

1. Pollack, R.: «Todos somos transexuales», World Media, El País, 12 de diciembre de 1996, p. 7. 
la vida? Parece como si los conceptos fundamentales de la biología fueran como resbaladizas anguilas que se libran de nosotros al tratar de asirlas con las manos del intelecto. En resumidas cuentas, en lo que llevamos descrito hemos generado tres grandes cuestiones que representan el núcleo central este apartado: ¿qué es la biología?, ¿qué es la vida? y ¿qué son los organismos?

En relación con la primera pregunta podemos decir que, para nosotros, "La biología no es la ciencia de la vida. La biología es la ciencia de los organismos». No es la ciencia de la vida porque el empleo del término vida no es científicamente adecuado, al carecer de un referente material. Sin embargo, su uso está profundamente arraigado en el lenguaje cotidiano y su empleo resulta útil. Creemos que en un sentido estrictamente científico se debería emplear el adjetivo viviente para acompañar las descripciones de las actividades y características de los organismos.

Respecto a la segunda pregunta, ¿qué es la vida?, podemos decir que no es ni una sustancia ni una cosa sino, mas bien, una función, un proceso o una propiedad. En este sentido Barcia Goyanes ${ }^{2}$ consideraba que la vida es una función que se realiza en el tiempo con intervención del organismo y del ambiente. Ya Letamendi ${ }^{3}$ analizó catorce definiciones diferentes de la vida y no encontró ninguna que resistiera la más ligera crítica. Por tanto, no existe una definición exacta de «algo" que podamos denominar vida. Como hemos dicho, el término vida es un concepto que se escapa del marco puramente científico para engrosar las filas de lo que el filósofo catalán Eugenio Trias ${ }^{4}$ denomina «pensamiento mágico». Utilizar el término vida en un contexto científico es, además de hacer pseudociencia, tratar de engañar a las gentes ingenuas vendiendo como científico lo que tan sólo es ideológico. Este tipo de argumentación «mágica» ha tenido y sigue teniendo especial difusión en muchas discusiones acerca de la legitimidad de diferentes prácticas biomédicas, relacionadas con los embriones. Se trata de ambientes en los que se vierten, como argumentos científicos, expresiones tan carentes de validez como "existe vida desde el momento de la concepción» (por supuesto, como existe "vida» en una bacteria o en una planta). Se puede leer un análisis serio de la ética relacionada con los embriones en un articulo reciente de Monika Bobbert 5 .

En tercer lugar, nos hemos preguntado ¿qué es un organismo? Con toda seguridad, los humanos somos los únicos organismos, de este planeta, con capacidad de hacerse esa pregunta. Si miramos a nuestro alrededor veremos muchos tipos de lo que nosotros denominamos objetos biológicos o vivientes. Nuestras mascotas, las plantas que adornan nuestros jardines, las que forman parte de

2. Cit. por Morales MACEDO, C.: Biología Fundamental, Barcelona, Salvat Editores, 1946, p. 24, en DE JUAN, J.: ¿De qué están hechos los organismos? El nacimiento de la mirada histológica, Alicante, Publicaciones de la Universidad de Alicante, 1999, p. 46.

3. Ibíd., p. 46.

4. TRIAS, E.: Metodología del pensamiento mágico, Barcelona, La Gaya Ciencia, 1970.

5. BOBBERT, M.: «Ethical questions concerning research on human embryos, embryonic stem cells and chimeras», Biotechnol. J, 1 (2006), pp. 1352-1369. 
nuestra dieta, las bacterias que nos infectan, ... todos ellos son organismos. Sin embargo y aunque el origen de este término se remonta a los debates entre el filósofo Leibniz y el médico y químico Stahl ${ }^{6}$, se han introducido otros términos para explicar las propiedades de los objetos biológicos o vivientes, frente a los objetos inertes, sin necesidad de recurrir al sustantivo vida. Se atribuye a Jacques Loeb, en 1916, la introducción del concepto de materia viviente ${ }^{7}$, al ser el primero en considerar la existencia de un tipo especial de materia, en contraste con la materia inerte. A partir de este momento empiezan a utilizarse ambos términos como contrapuestos.

Nuestro Universo tiene la friolera de 13.700 millones de años, desde que una gran explosión, conocida como el big bang, hiciera posible su aparición, según nos han hecho saber eminentes científicos del ámbito de la física. Hace 4.600 millones de años se formó nuestro planeta, a partir de una densa nube de gas y polvo, y 1000 millones más tarde, o sea, hace 3600 millones de años, hicieron su aparición los primeros organismos de la Tierra, dando lugar a ese fenómeno que muchos denominan «el origen de la vida».

Si tenemos en cuenta la teoría de la evolución, y nosotros la tenemos, en el tránsito de la materia inerte a los organismos debió de existir alguna forma intermedia de la materia viviente. Para soslayar esta dificultad, Orgel ${ }^{8}$ introdujo el concepto de CITROENS, palabra que se refiere a las siglas de Objetos Complejos Transformadores de Información y Reproducibles que Evolucionan por Selección Natural. En opinión de Orgel todo lo que sea un CITROENS puede ser considerado materia viviente. En este sentido son CITROENS todos los objetos vivientes existentes, las moléculas relacionadas con los ácidos nucleicos (DNA, RNA, ribozimas, elementos transponibles, plásmidos, viroides, virus, etc.) u otras moléculas complejas, con las mencionadas características. Estas moléculas pudieron ser las primeras manifestaciones de la materia viviente, antes de la aparición de los primeros organismos. Es quizá en este apartado donde podríamos incluir objetos biológicos tan interesantes como los priones (nombre derivado de partícula proteinacea infecciosa), descubiertos por el Premio Nobel de Medicina Stanley Prusiner ${ }^{9}$ en 1982 y causantes de la encefalopatía espongiforme bovina o "enfermedad de la vacas locas" y de la enfermedad de Creutzfeldt-Jacob en los humanos. Finalmente, mencionaremos las moléculas autorreplicantes, moléculas sintéticas derivadas de la mezcla de otras que producen compuestos,

6. Wolfe, C.T.: «The concept of organism, an historical and conceptual critique». Artículo presentado en julio de 2005 en el ISHPSSB Meeting en la Universidad de Guelph, Ontario, dentro del Workshop titulado «Naturalizing Teleology?» (http://people.bu.edu/teleolog/materials/ ORGANISM\%20Guelph.pdf). Existe una versión más extensa, del año 2004, publicada en francés en Multitudes 16, con el titulo "La catégorie d'«organisme» dans la philosophie de la biologie. Retour sur les dangers du réductionnisme» (http://multitudes.samizdat.net/article. php3?id article $=1370$ ).

7. De JUAN, J.: Op. cit., p. 47.

8. ORGEL, L.E.: Los orígenes de la vida, Madrid, Alianza Universidad, 1975, pp. 193-202.

9. PRUSINER, S.B.: "Novel proteinaceous infectious particles cause scrapie», Science, 216 (1982), pp. 136-144. 
capaces de autorreplicarse en el laboratorio, como ha puesto de manifiesto el profesor Rebeck ${ }^{10}$ del Massachussets Institute of Technology (MIT).

La materia viviente, a diferencia de lo que ocurre con la inerte, no se encuentra repartida de un modo difuso en la Biosfera. Por el contrario, se nos presenta como sistemas polimoleculares, de estructura compleja y muy bien delimitados e individualizados, es decir, formando organismos. A pesar de que los objetos biológicos que consideramos organismos son claramente discretos y aislados, tal separación se hace difícil cuando tratamos de clasificarlos en un número reducido de grupos, a fin de no perdernos en la abrumadora cifra de especies que se conocen actualmente en la Biosfera, entre 1,5 a 5 millones $^{11}$.

Ordenar las cosas del universo en grupos o clases es fundamental para nuestro entendimiento de la naturaleza. El término clase natural fue introducido por John Stuart Mill ${ }^{12}$ e incorporado en la filosofía contemporánea por Quine en su ensayo "Natural Kinds»". Una clase natural («natural kind»), es un conjunto ontológicamente real, es decir, no resultante de la agrupación artificial de elementos, elaborada por uno o varios sujetos humanos, en virtud de criterios subjetivos. Las clases naturales son, por consiguiente, agrupaciones de objetos o de individuos reunidos de forma natural. Tradicionalmente se denominan así aquellas agrupaciones que siguen las leyes de la naturaleza, como por ejemplo los minerales, las plantas, los animales, etc. Hay mucha controversia acerca de qué objetos biológicos deben considerarse categorías naturales. Así, de términos como especie, organismo, raza, sexo y orientación sexual (la mayoría de los objetos analizados aquí) es controvertido decidir si son categorías naturales o no. Lo que sí que parece claro es que los productos culturales (artefactos culturales) no entran dentro de ese grupo ${ }^{14}$. Cualquier conjunto de objetos forman una clase natural si y sólo si es "proyectable», es decir, si las proposiciones acerca de algún miembro de ese conjunto pueden ser extendidas, científicamente, por razonamiento inductivo, a otros miembros. En general, podemos decir que las distintas culturas realizan análogas clasificaciones naturales ${ }^{15}$. En biología sistemática, tanto la especie como el organismo son categorías naturales que tienen sus propias reglas, a diferencia de los individuos que las componen ${ }^{16}$.

Aunque a simple vista el concepto de organismo parece claro, sin embargo, no ha estado exento de controversia. En efecto, en el año 1989, la revista «American

10. ReBECK, J.: «Moléculas sintéticas autorreplicantes», Investigación y Ciencia (septiembre 1994), pp. 20-26.

11. MAY, R.M.: "Número de especies que habitan la Tierra», Investigación y Ciencia (diciembre 1992), pp. 6-12.

12. GHISELIN, M.T.: «Natural kinds and supraorganismal individuals», en Douglas L. Medin y Scott Atran (eds.): Folkbiology, Boston, The MIT Press, 1999, pp. 447-460.

13. Quine, W.V.O.: "Natural Kinds», en Ontological Reality and Other Essays, New York, NY, Columbia University Press, 1969, pp. 114-138.

14. GHISELIN, M.T.: Op. cit.

15. Molino, J.: "Toward an Evolutionary Theory of Music and Language», The Origins of Music, Cambridge, Mass, A Bradford Book, The MIT Press, 2000

16. GHISELIN, M.T.: Op. cit. 
Zoologist» planteó la siguiente pregunta: ¿Existen los organismos? ${ }^{\text {17. }}$. La pregunta no era baladí debido a que el gran avance de la biología molecular llevó el conocimiento biológico a un reduccionismo extremo. Tres son los principales tipos de respuesta que se han dado, a lo largo de la historia, a la pregunta de Michael Ruse: vitalista/emergentista, reduccionista e instrumentalista ${ }^{18}$. Para los reduccionistas, los organismos son conjuntos de átomos que se rigen por las leyes de la física, y de la química, y punto y fina ${ }^{19}$. Dicho de otro modo, los organismos «no son entidades reales", lo único real son las entidades descritas por la física y la bioquímica. Un caballo, una margarita no existen más que por una convención verbal o social. Según Alexander Rosemberg ${ }^{20}$, los organismos tan sólo son términos convencionales. Para los vitalistas/emergentistas, un organismo no se reduce a ser un mero mecanismo, una máquina con múltiples piezas y funciones. Para ellos, son entidades con nuevas propiedades emergentes. Finalmente, la corriente instrumentalista es la que aporta la respuesta más interesante. En efecto, evita los escollos del vitalismo y del mecanicismo, pero retiene lo mejor de ambas corrientes: el deseo de explicar un sistema complejo y sus transformaciones temporales, y el poder explicativo del reduccionismo. Para ellos, el organismo es la proyección de una categoría real (Clase natural) con fines pragmáticos. Son complejos conjuntos de niveles de organización, colocados según patrones fuertemente ordenados, sin que ninguno de estos niveles sea más importante que los otros, para un completo entendimiento de los fenómenos vitales ${ }^{21}$. Actualmente parece que de nuevo se esté produciendo un resurgir de las teorías holistas sobre el concepto de organismo 22 .

Para nosotros, los organismos, incluidos los humanos, "somos» conjuntos ontológicamente reales (clases naturales). La esencia de ser organismos nos la da el hecho de poseer cuerpos con ciclos vitales ${ }^{23}$. Somos cuerpos cambiantes, a lo largo del tiempo, con un destino que depende de la interacción entre nuestros programas de fábrica y la acción del medio. Para Dennett ${ }^{24}$ los humanos somos criaturas con inmensas capacidades de predicción y reflexión sobre nuestro futuro que nos diferencian, radicalmente, de los demás organismos. Somos el resultado, básicamente, de la dinámica de potenciación debida, en parte, a nuestro lenguaje. En la vida cotidiana también utilizamos el término organismo

17. RUSE, M.: «Do Organisms Exist?», Amer Zool, 29 (1989), pp. 1061-1066.

18. Wolfe, C.T.: «The concept..., op.cit; WolfE, C.T.: "L'organisme, une fiction instrumentale», Sciences et Avenir. Hors-Série (Juillet/Aout, 2006), p. 41 (cita 6).

19. Hull, D.: «Philosophy and biology», en G. Fløistad (ed.): Contemporary philosophy: a new survey, The Hague, M. Nijhoff, 2 (1981), p. 282.

20. Cit. por Wolfe, C.T.: «L'organisme..., op. cit., p. 41.

21. BoCK, W.: "Organisms as Functional Machines: A Connectivity Explanation", American Zoologist, 29 (1989), pp. 1119-1132.

22. LAUBICHLER, M.: "The Organism is dead. Long live the organism!», Perspectives on Science 2000, 8 (2000), pp. 286-315.

23. BOnNeR, J.T.: «El ciclo vital», en Ciclos Vitales. Confesiones de un biólogo, Madrid, Alianza Universidad, 1995, p. 31. Como señala John, Tyler Bonner en este libro, los organismos no son solamente adultos: son ciclos vitales.

24. DenneTT, D.C.: Darwin's dangerous idea, New York, A Touchtone Book, 1996. 
para referirnos al cuerpo ya que, como también dice Dennett ${ }^{25}$, «todo lo vivo tiene un cuerpo». En este sentido, hablar del cuerpo sería un importante apartado a considerar, en el tema que nos ocupa, pero su inclusión en este artículo se aleja de nuestros objetivos.

Finalmente, si tuviéramos que extraer una serie de conclusiones generales que sinteticen las principales ideas expuestas en este apartado, éstas podrían ser:

1) Los conceptos científicos de la Biología moderna no son estructuras monolíticas y estables. Están tremendamente influidas por la cosmovisión (ideología, filosofía, cultura, creencias, etc.) que cada uno de nosotros tiene. Podemos decir que el nacimiento de nuevos conceptos, siempre, ha estado más influido por el ámbito cultural y las concepciones preexistentes que por los propios hechos científicos.

2) El objeto material de la Biología son los organismos, no la vida. Es decir, el estudio de esa clase natural de objetos caracterizados por poseer un cuerpo, sometido a un ciclo vital, cuyo comportamiento depende de la interacción de sus propios programas con el ambiente. El objeto formal de la Biología será el estudio, en particular, de esos cuatro elementos: cuerpo, ciclo vital, programas y ambiente. Los organismos humanos participamos de las mismas características.

3) El término vida es un concepto extra-científico, un concepto «maná». La vida como sustantivo, como esencia, no existe. Utilizar el concepto de "vida» como un concepto científico para argumentar, a favor o en contra, de determinadas prácticas sociales (aborto, eutanasia, contracepción, etc.) es inadmisible científicamente.

\section{CONCEPTO DE SEXO Y DE SEXUALIDAD}

Para abordar de forma rigurosa los temas relacionados con el concepto de género, es importante tener claros los conceptos de sexo, identidad de género, papel de género y orientación sexual, que desarrollaremos en este y en los siguientes apartados. Aquí, reflexionaremos sobre el concepto de sexo desde una perspectiva biológica.

Una de las características más importantes de los términos sexo, sexualidad o experiencia sexual es su gran ambigüedad, muy relacionada con el contexto en el que se utilizan. En efecto, como dicen Diamon y Yates ${ }^{26}$, esos términos pueden hacer referencia tanto al coito, como a las características genéticas y biológicas de un determinado individuo. De ahí que comenten, con cierta ironía, que la ambigüedad del término sexo, no se debe atribuir sólo a Bill Clinton al no considerar como tal, ante un tribunal, los contactos orogenitales. El 50\% de los adolescentes opinan igual.

25. DenneTT, D.C.: Tipos de mente, Madrid, Editorial Debate, 2000.

26. Diamon, M. y Yates A.: «Preface: Sex and gender», Child Adolesc Psychiatric Clin N Am, 13 (2004), pp. Xv-xvii. 
Sin embargo, desde el punto de vista de la Biología, se denomina sexo al conjunto de características biológicas de un organismo que permiten diferenciarlo como portador de uno u otro tipo de células reproductoras o gametos (óvulos o espermatozoides), o de ambos (organismos hermafroditas). Sexualidad, por su parte, se refiere a las actividades que los organismos llevamos a cabo para poder intercambiar nuestro material genético y conseguir una mayor diversidad y adaptación al medio. Entre los organismos animales existen dos grandes variedades atendiendo al origen de sus gametos ${ }^{27}$ : a) organismos gonocóricos (con dos tipos de individuos, atendiendo a sus gametos y características sexuales). En ellos podemos hablar de dos sexos, uno portador de los gametos masculinos $\mathrm{y}$ otro portador de los gametos femeninos, $\mathrm{y}$ b) organismos hermafroditas que son portadores de ambos gametos y por lo tanto de ambos sexos (hermafroditas verdaderos o sincrónicos). En los peces ${ }^{28}$ la forma más frecuente de hermafroditismo es el secuencial, debido a que sus dos gónadas, masculina y femenina, no maduran al mismo tiempo. En unos casos, maduran antes las gónadas masculinas que las femeninas (hermafroditismo protándrico), como ocurre en la dorada (Sparus auratus) que comienza como macho y posteriormente se hace hembra, mientras que en otros maduran antes las gónadas femeninas que las masculinas (hermafroditismo protoginico), como en el mero (Epinephelus guaza). Parece ser que el cambio de sexo, en estos casos, está determinado fundamentalmente por razones "sociales».

En el caso de nuestra especie, como organismos gonocóricos que somos, estamos acostumbrados a hablar de dos sexos, masculino (hombre) y femenino (mujer), así como de una serie de características que nos permiten establecer diferencias entre ambos. Los caracteres sexuales fueron definidos en el año 1869 por el anatomista Hunter ${ }^{29}$ quien los dividió en primarios, presentes ya al nacer, y secundarios, los que se adquieren con el desarrollo sexual en la pubertad. Más tarde, Havellock Ellis ${ }^{30}$, padre de la sexología moderna, los clasificó en primarios, las gónadas (ovarios o testículos), secundarios, el tracto genital masculino (pene, próstata, conductos seminíferos, epidídimo, conducto deferente y vesículas seminales) y femenino (vulva, vagina, útero y trompas) y terciarios, la morfología corporal, la voz, el psiquismo, y el esqueleto, que sólo se alcanzan en la pubertad. Más recientemente, los caracteres sexuales de los humanos han sido divididos en los siguientes tipos ${ }^{31}$, con alguna matización nuestra, basada en otros autores:

27. De Juan, J.; García-Irles, M.; Pérez-CañaVeras, R.M. y Mengual, R.: «Influencia del sistema visual en la reproducción de los peces», en S. Zamora, B. Agulleiro y P. García (eds.): Acuicultura I: Biología Marina. Reproducción y desarrollo, Murcia, Universidad de Murcia, pp. 191-217.

28. Ibíd.

29. HUNTER, J.: Essays and observations, Londres, 1869.

30. ElLIS, H.: Man and Woman, Boston, Houghton and Miffin, 1929.

31. BOTELLA LLUSIA, J.: «La evolución de la sexualidad y los estados intersexuales: a los sesenta y siete años de un libro fundamental» y "Los caracteres sexuales y su clasificación", en J. Botella Llusiá y A. Fernández de Molina (eds.): La evolución de la sexualidad y los estados intersexuales, Madrid, Editorial Díaz de Santos, 1997, pp. 1-11 y 77-84. 
a) Primarios o genéticos, que se encuentran contenidos en los genes (sexo genético) y en los cromosomas de todas las células del cuerpo (sexo cromosómico, según la clasificación de Migeon y Wisniewski) ${ }^{32}$. Son el par de cromosomas denominados sexuales (XX para la mujer y XY para el hombre) y los genes implicados, como el gen SRY (Sex-determining gene region of the $Y$ chromosome), ubicado en el cromosoma Y del varón.

b) Secundarios (Sexo gonadal, de la clasificación de Migeon y Wisniewski), consistentes en la presencia de los ovarios, con sus óvulos y hormonas esteroideas femeninas (estrógenos y progesterona), en la mujer, y de los testículos, con sus espermatozoides y hormonas esteroideas masculinas (andrógenos), en el hombre. Bajo el nombre de sexo hormonal, Migeon y Wisniewski denominan al tercer parámetro de su clasificación. Precisamente, las mencionadas hormonas son las responsables del desarrollo y normal funcionamiento del resto de los caracteres sexuales y del organismo en general. En el caso de los andrógenos, no sólo basta con su producción, sino que es necesaria la presencia de receptores para ellos, en las células de los genitales externos. Un factor importante en el desarrollo sexual es el denominado Factor Determinante del Testículo (TDF, siglas de testis determining factor) identificado por Vilain y $\mathrm{McCabe}^{33}$. Este factor se localiza en el brazo corto del cromosoma $\mathrm{Y}^{34}$, donde se identificó el mencionado gen SRY. El testículo también es responsable de la producción de una proteína, la sustancia inhibidora del conducto de Müller, implicada en la diferenciación sexual masculina, especialmente en la secuencia de eventos que determinan la regresión de los conductos de Müller y la no formación del útero, trompas de Falopios y tercio superior de la vagina.

c) Terciarios o genitales, es decir las características diferenciales que existen en el tracto genital femenino (trompas de Falopio, útero, vagina y vulva) y en el masculino (epidídimo, conducto deferente, vesículas seminales, próstata y genitales externos). En la clasificación de Migeon y Wisniewski todas estas características, excepto los genitales externos, se recogen como el cuarto parámetro de la biología del sexo, bajo el nombre de estructuras reproductivas internas. Para los citados autores, los genitales externos representan el quinto parámetro a considerar al caracterizar el sexo.

d) Cuaternarios o aparentes, formados por todas aquellos rasgos y características somáticas que a primera vista nos evocan feminidad o masculinidad, tales como: desarrollo de las mamas, distribución de la grasa y del vello, forma del esqueleto, desarrollo muscular, características de la voz, características y textura de la piel, etc.

32. Migeon, C. y WisnieWSKI, A.: «Sexual differentiation: From genes to gender», Horm Res, 50 (1998), pp. 245-251.

33. VILAIN, E. y MCCABE, E.: "Mammalian sex determination: From gonads to brain», Mol Genet Metabol, 65 (1998), pp. 74-84.

34. Haqa, C. y DonaHOE, P.: «Regulation of sexual dimorphism in mammals», Physiol Rev, 78 (1998), pp. 1-33. 
e) De quinto orden o psíquicos (gender-identity/role) que para Botella Llusiá estarían representados por la libido, el instinto de pareja, diferencias neurobiológicas, el instinto maternal, etc. Características que, en nuestra opinión, son meras especulaciones. En la clasificación de Migeon y Wisniewski, constituyen el sexto parámetro, bajo el nombre de «cerebro sexual» (Brain Sex).

Aunque con este listado de características sexuales parecería casi imposible que ningún ser humano pudiera caer fuera de las categorías biológicas de hombre o mujer, sin embargo existen situaciones de difícil catalogación recogidos bajo el nombre de Trastornos del Desarrollo Sexual o DSD o DSDs en plural ${ }^{35}$, según su abreviatura en inglés (Disorders of Sex Development). Se trata de una terminología utilizada para desplazar términos como "intersexo", "tercer sexo", "pseudohermafroditismo", "hermafroditismo", "sex reversal», etc. porque son considerados, con frecuencia, peyorativos u ofensivos para las personas portadoras de estas situaciones ${ }^{36}$.

Los DSDs se definen como alteraciones congénitas en las que el desarrollo de los caracteres sexuales (primarios, secundarios, etc.) es atípico ${ }^{37}$. Se trata de un heterogéneo grupo de condiciones que interfieren con la determinación y diferenciación del sexo, durante el desarrollo embrionario y fetal. Los datos estadísticos sobre la prevalencia de estos procesos son limitados ${ }^{38}$. Se estima que hay un caso por cada 5.500 nacidos $^{39}$, siendo la hiperplasia adrenal congénita o CAH (siglas de Congenital Adrenal Hyperplase) el trastorno más común como causa de genitales ambiguos, representando aproximadamente el $50 \%$ de todos los casos de ambigüedad genital del periodo neonatal ${ }^{40}$. A nivel mundial, la incidencia de CAH es de 1:15.000 aunque varia considerablemente en diferentes poblaciones ${ }^{41}$. La segunda causa más frecuente de DSDs es la Disgenesia Gonoadal Mixta ${ }^{42}$ con una incidencia de 1:10.000.

35. NABHAN, Z.M. y LeE, P.A.: "Disorders of sex development», Curr Opin Obstet Gynecol, 19 (2007), pp. 440-445.

36. NABHAN, Z.M. y LeE, P.A.: «Disorders of sex..., op. cit. Ver también ConN, J.; GILLAM, L. y CONWAY, G.: "Revealing the diagnosis of androgen insensitivity syndrome in adulthood», $B M J$, 331 (2005), pp. 628-630.

37. NABHAN, Z.M.y LEE, P.A.: «Disorders of sex..., op. cit.

38. Ibíd. Ver también Blackless, M.; Charuvastra, A.; Derryck, A.; Fausto Sterling, A.; LaUZZANNE, K y LeE, E.: «How sexually dimorphic are we? Review and síntesis», Am J Hum Biol., 13 (2000), pp. 260-268.

39. NABHAn, Z.M. y Lee, P.A.: "Disorders of sex ..., op. cit. Ver también SAX, L.: "How common is intersex? A response to Anne Fausto-Sterling», J Sex Res, 39 (2002), pp. 174-178.

40. NABHAN, Z.M. y LEE, P.A.: «Disorders of sex..., op. cit. Ver también THYEN, U.; LANZ, K.; HOLTERHUS, P.M. y HIORT, O.: "Epidemiology and initial management of ambiguous genitalia at birth in Germany», Horm Res, 66 (2006), pp. 195-203.

41. NABHAN, Z.M. y LEe, P.A.: "Disorders of sex..., op. cit. Ver también PanG, S.Y.; WALlace, MA.; Hofman L.; Thuline, H.C.; DORChe, C.; LlyiOn, I.C.; DobBins, R.H.; KlinG, S.; FujiedA, K. y SuWA, S.: «Worldwide experience in newborn screening for classical congenital adrenal hyperplasia due to 21-hydroxylase deficiency", Pediatrics, 81 (1988), pp. 866-874.

42. NABHAN, Z.M. y LeE, P.A.: "Disorders of sex ..., op. cit. Ver también SKAKKEBAEK, N.E.: "Testicular dysgenesis síndrome», Horm Res, 60 (Suplem.3) (2003), p. 49. 
Del análisis de estos casos, la bióloga celular y del desarrollo, Ann Fausto Sterling, en sendas publicaciones con los títulos de The five sexes (1993) y The five sexes, revisited $(2000)^{43}$ elaboró una clasificación de los sexos en las siguientes cinco categorías:

a) «Male»: Hombres con el aparato reproductor normal.

b) «Female»: Mujeres con el aparato reproductor normal.

c) «Herms»: Hermafroditas verdaderos, nacidos con tejido testicular y ovarico

d) "Merms»: Seudohermafroditas masculinos, nacido con testículos y algún aspecto femenino de sus genitales.

e) «Ferms»: Seudohermafroditas femeninos, nacido con ovario y algún aspecto masculino de sus genitales.

No es éste es el lugar para describir los mecanismos que determinan las mencionadas modificaciones. Los interesados en el tema pueden leer el capítulo 16 del libro de Bruce M. Carlson ${ }^{44}$, donde encontrarán una clara explicación del desarrollo normal de los sistemas reproductores y de sus alteraciones.

Finalmente, debemos decir que los casos de organismos con modificaciones en el desarrollo que dan lugar a situaciones intersexuales no son exclusivos de la especie humana. Un ejemplo muy interesante y llamativo se da en el pajarillo pinzón zebra (Taeniopygia guttata) debido a su librea rallada ${ }^{45}$. Se trata de un caso raro de ejemplares ginandromórficos, caracterizados porque la mitad derecha de su cuerpo presenta características sexuales de macho (librea muy vistosa, testículo, cerebro cuyas células poseen cromosomas y genes machos), mientras que el lado izquierdo tiene un aspecto típicamente de hembra (librea discreta, ovario, neuronas con cromosomas sexuales característicos de la hembra).

\section{IDENTIDAD DE GÉNERO Y PAPEL DE GÉNERO}

Otras palabras que generan confusión son las de género e identidad. Como con el término sexo, la palabra género tampoco está exenta de usos e interpretaciones ambiguas. En ocasiones se emplea como sinónimo de sexo, otras veces se utiliza para designar un constructo social, en el que se separa lo sociológico de lo biológico y por último, es considerada como un elemento meramente lingüístico. El término identidad hace referencia a "como se considera uno así mismo»: ¿como hombre o como mujer?; ¿como homosexual, heterosexual o bisexual?, ¿como optimista o pesimista?, etc.

43. Fausto Sterling, A.: "The five sexes», The Sciences (March/August 1993), pp. 20-24; Fausto STERLING, A.: "The five sexes, revisited», The Sciences (July/August 2000), pp. 19-23.

44. CARLSON, B.M.: "Sistema urogenital», en Embriolgía humana y biología del desarrollo, Madrid, Mosby/Elsevier, pp. 393-427.

45. Agate, R.J.; Grisham, W.; Wade, J.; Mann, S.; Wingfield, J.; Schanen, C.; Palotie, A. y ARNOLD, A.P.: "Neural, not gonadal, origin of brain sex differences in a gynandromorphic finch", PNAS, 100 (2003), pp. 4873-4878. 
El término identidad de género (Gender Identity) fue introducido por Hooker ${ }^{46}$, un psicólogo y Stoller ${ }^{47}$, un psicoanalista, casi simultáneamente, a principios de los sesenta. Para John Money ${ }^{48}$ la identidad de género es la propia categorización de una individualidad como hombre, mujer o ambivalente, en la que uno tiene autoconciencia de los propios procesos mentales y de las conductas propias. Aquí entendemos la identidad de género como la conciencia de sentirse hombre o mujer ${ }^{49}$

El papel de género (Gender Role) es la manifestación pública de la propia identidad de género, lo que uno dice y hace, en base a que se infiera que es un hombre o una mujer o se corresponde con una u otra de esas categorías ${ }^{50}$. Son los rasgos de conducta, actitud y personalidad que una sociedad, en un determinado periodo histórico y cultural, designa como masculino o femenino. Lo que esa sociedad "espera» de una persona con un determinado sexo o adscrita a un determinado género, en el "entendimiento» de que a cada sexo/género se le atribuye, cultural y socialmente, un determinado comportamiento (preferencia por unos juguetes u otros, manierismo, estilo de vestir, etc. $)^{51}$. Las humanidades y las ciencias sociales definen el papel de género como un conjunto de normas de conducta asociadas a hombres y mujeres, respectivamente, dentro de un determinado grupo o sistema.

Íntimamente relacionado con la identidad de género se encuentra el transexualismo o transexualidad. Básicamente consiste en desarrollar una identidad de género que no es acorde con los caracteres sexuales comentados más arriba (primarios, secundarios, etc.). Algunos han definido el transexualismo como una forma extrema de disforia de género. Por disforia de género se entiende la discrepancia entre identidad/papel de género, por una parte, y las características físicas del cuerpo, por otra ${ }^{52}$. Para otros consiste en el disconfort resultante de vivir como hombre o como mujer ${ }^{53}$. Cuando el distress producido es muy intenso, solicitan el cambio de sexo. En el transexualismo, la identidad/papel de un sexo coexisten con los caracteres sexuales (primarios, secundarios, etc.) del otro sexo,

46. MONEY, J.: "The conceptual neutering of geneder and the criminalization of sex», Arch Sex Behav, 14 (1985), pp. 279-290.

47. StOlLeR, R.: «The hermafroditic identity of hermafrodites», J Nerv Ment Dis, 139 (1964), pp. 453-457.

48. MONEY, J.: «The development of sexuality and eroticism in humankind, Q Rev Biol., 56 (1981), pp. 379-404; MONEY, J.: The sexological concept of bipotentiality, Exp Clin Endocrin, 98 (1991), pp. 151-153.

49. NABHAn, Z.M. y LeE, P.A.: «Disorders of sex ..., op. cit. MOneY, J. y EHRHARDTT, A.A.: Man and women, boy and girl, Baltimore MD, Johns Hopkins University Press, 1972; BYNE, W.: «Developmental endocrine influences on gender identity: implications for management of disorders of sex dvelopment», Mt Sinai J Med, 73 (2006), pp. 950-959.

50. GOOREN, L.: "The Biology of human psychosexual differentiation», Hormones and Behavior, 50 (2006), pp. 589-601.

51. NABHAN, Z.M. y LeE, P.A.: «Disorders of sex ..., op. cit. MONEY, J. y EHRHARDTT, A.A.: Op.cit.; BYNE, W.: Op.cit,. Ver cita 48.

52. GOOREN, L.: "The Biology of human..., op. cit.

53. GreEN, R.: «Gender development and reassignment», Psychiatry, 6 (2007), pp. 121-124. 
dentro de una misma persona. Por otra parte, con las técnicas de evaluación biológica actuales, no se observan signos objetivos de DSDs en las personas transexuales, por lo que el transexualismo precisa como requisito que no haya historia clínica de DSDs. En resumen, la persona transexual considera que su identidad/papel de género es suyo, pero se sienten atrapados en un cuerpo que no es el suyo. Por ello, podemos decir que la transexualidad no es una disforia de género, sino una disforia de cuerpo.

La prevalencia es de 1:10.000 casos de transexualidad de hombre a mujer (MtF, siglas de Male, transexuality y Female) y de 1:30.000 para la variante de mujer a hombre (FtM). La identidad y la conducta de género emergen tempranamente en la vida de las personas, concretamente hacia el final del tercer año. Hay madres que lo detectan cuando los bebes empiezan a hablar e insisten en utilizar sus vestidos y zapatos, o al mostrar interés sólo por los juguetes de niñas. Por otra parte, no todos los niños con discrepancias de género se convierten en transexuales. Solamente un $23 \%$ de los casos con un problema de género en la niñez pasan a ser transexuales cuando adultos ${ }^{54}$. Para mayor información sobre transexualidad puede consultarse la obra de Becerra ${ }^{55}$.

\section{ORIENTACIÓN SEXUAL}

La orientación sexual se refiere a las conductas derivadas de las preferencias o respuestas eróticas que cada uno tiene, y que incluyen desde conductas explícitas, tales como las descritas por Byne ${ }^{56}$, androfilica (atracción por el hombre), ginefilica (atracción por la mujer) o bisexual (atracción por ambos), fantasías sexuales y atracción sexual, de ahí que se hable también de heterosexualidad, homosexualidad y bisexualidad ${ }^{57}$. También se puede definir como la respuesta dada por una persona a un estimulo sexual. Fausto Sterling en su libro Cuerpos sexuados ${ }^{58}$, habla de otros términos como ebofilia (preferencia por los jóvenes púberes), efebofilia (preferencia por los varones adolescentes), pedofilia (preferencia por los niños), ginofilia (preferencia por las mujeres adultas) y androfilia (preferencia por varones adultos). De todas las respuestas sexuales, la más relevante para definir la orientación sexual es el sexo de la persona por la que un sujeto siente atracción ${ }^{59}$. Sin embargo, el término preferencia sexual es probablemente poco acertado ${ }^{60}$.

54. COHEn-KetTenis, P.T. y GoOREn, L.J.G.: «Transsexualism: a review of etiology, diagnosis and treatment», Journal of Psychosomatic Research, 46 (1998), pp. 315-333. COOLIDGE, F.L.; THEDE, L.L, YOUNG, S.E.: "The heritability of gender identity disorder in a child and adolescent twin sample», Behavior Genetics, 32 (2002), pp. 251-257.

55. BeCERrA Fernández, A. Transexualidad: La búsqueda de una identidad, Madrid, Díaz de Santos, 2003.

56. BYNE, W.: "Developmental endocrine influences..., op. cit.

57. NABHAN, Z.M. y LEE, P.A.: «Disorders of sex..., op. cit. MONEY, J. y EHRHARDTT, A.A.: Op.cit.; BYNE, W.: Op. cit. Ver cita 48.

58. FAusto Sterling, A.: Cuerpos sexuados, Barcelona, Editorial Melusina, 2006, p. 21.

59. ZUCKER, K.J.: «Intersexuality and geneder identity differentiation», J Pediatr Adolesc Gynecol, 15 (2002), pp. 3-13.

60. GOOREN, L.: «The Biology of human..., op. cit. 


\section{SEXO, GÉNERO Y NEUROCIENCIAS}

En este último apartado nos enfrentamos a un tema, a la vez que apasionante, desde el punto de vista científico, altamente controvertido, tanto desde la perspectiva científica como de la ideológica. En el número 1 de Feminismo/ $\mathrm{s}^{61}$ hay un excelente estudio en el que se analizan los prejuicios que, sobre la mujer, han existido y existen en el ámbito de la ciencia. Nuestro interés aquí es dar una visión general y aportar datos sobre el tema y que cada uno extraiga sus propias conclusiones.

\subsection{De la teoría de la neutralidad psicosexual a las teorías biológicas}

El desarrollo psicosexual humano es un proceso complejo, influido por múltiples factores, a saber: genéticos, gonadales, hormonales, desarrollo, neurobiológicos, culturales, sociales y familiares ${ }^{62}$. Tres son los aspectos a considerar en este proceso: la identidad de género, el papel de género y la orientación sexual.

En los años sesenta, debido a los avances de la cirugía plástica y de la teoría de la identidad de género al uso, que consideraba el género como un producto de los genitales y de la educación ("genitales más crianza»), se impuso la cirugía correctiva de los órganos sexuales, en recién nacidos con DSDs. Esta práctica se viene realizando, desde entonces, con una proporción de cerca de una intervención por cada 2000 nacimientos. En la mayoría de los casos se reconstruyen los genitales como femeninos, reasignando al bebé con DSD como niña. Esta preferencia quirúrgica se debe a su mayor facilidad, más que a consideraciones sobre el futuro de la criatura.

La base de estas prácticas quirúrgicas es la creencia de que al nacer todos somos psicosexualmente neutros, en cuanto a la identidad de género ${ }^{63}$. De ahí que, para algunos autores ${ }^{64}$, la educación recibida fuera mejor predictor, tanto de la identidad y del papel de género como de la orientación sexual, que las variables biológicas. Dicho de otro modo, para esos autores, en el desarrollo psicosexual predominaba lo adquirido o ambiental (nurtura) sobre lo congénito o heredado (natura). Podemos imaginar las graves consecuencias que la «teoría de la crianza y socialización», unida a las prácticas quirúrgicas correctivas, han tenido sobre las numerosas personas sometidas a ellas. En las últimas dos décadas, la creencia en la influencia ambiental y en la maleabilidad temprana del género ha sido puesta

61. Miqueo, C.; Barral Morán, M.J.; Delgado EcheVarría, I.; Fernández-Turrado, T. y MAGaLlón, C.: Del análisis crítico a la autoridad femenina en la ciencia», Feminismo/s, 1 (2003), pp. 195-215.

62. NABHAN, Z.M. y LEE, P.A.: «Disorders of sex..., op. cit. BYNE, W.: «Developmental endocrine influences..., op. cit.

63. NABHAN, Z.M. y LEE, P.A.: «Disorders of sex ..., op. cit.; DiAmOND, M.A.: «A critical evaluation of the ontogeny of human sexual behavior», Quarterly Rev Biol., 40 (1965), pp. 147-176.

64. MONEY, J.; HAMPSON, J.G. y HAMPSON, J.L.: «Imprinting and the stablishment of gender role», AMA Arch Neurol Psychiatry, 77 (1957), pp. 333-336. 
en duda de forma contundente ${ }^{65}$, a la luz de numerosos y nuevos trabajos de investigación, de cuyos datos hablaremos en lo que sigue. En consecuencia, está surgiendo todo un cuerpo de evidencias que indican que las diferencias en la identidad de género, en el papel de género y en la preferencia sexual, tienen gran parte de su sustrato en el propio cerebro ${ }^{66}$.

\subsection{Factores que influyen en el desarrollo del Sistema Nervioso (SN)}

A partir de la sexta semana de vida intrauterina, las gonadas (ovarios o testículos) comienzan a desarrollarse, al ponerse en marcha una cascada de genes, como el gen SRY, ubicado en el cromosoma Y del padre. Una vez diferenciadas, las gonadas comienzan a producir sus respectivas hormonas sexuales (testosterona, estrógenos y progesterona). La producción de testosterona y dihidrotestosterona, por los testículos embrionarios del niño, es necesaria para el desarrollo de sus órganos sexuales entre la 6 y la 12 semanas del embarazo. La conversión de la testosterona periférica en dihidrotestosterona resulta esencial para la formación de los órganos sexuales en el feto masculino, especialmente del pene, la próstata y el escroto. En el caso de los embriones de niña, el desarrollo de los genitales externos se produce, básicamente, por la ausencia de andrógenos ${ }^{67}$. Una vez producida la diferenciación de los órganos sexuales, como masculinos o femeninos, la siguiente etapa es la diferenciación del cerebro embrionario.

Durante la vida intrauterina, el SN está inmerso en un ambiente influido por las hormonas sexuales circulantes mencionadas, que contribuyen a la organización de sus neuronas, sinapsis y circuitos. Al llegar la pubertad, los circuitos cerebrales se activan por acción de las mismas hormonas, ya que desde el comienzo del desarrollo del cerebro, hasta la edad adulta, son muchas las neuronas de diferentes localizaciones del SN que poseen receptores para las hormonas sexuales. En la diferenciación femenina del cerebro de las niñas parece ser que éste produce alfa-fetoproteína (AFP) que se une fuertemente a los estrógenos maternos circulantes, para protegerse de sus efectos masculinizantes ${ }^{68}$. Sin embargo, en la diferenciación masculina, al no haber estrógenos, es el propio cerebro el que es capaz de producirlos. De esta manera, la testoterona del niño amplia su capacidad de acción una vez convertida en estrógenos por acción de la enzima denominada aromatasa.

65. DiAmOND, M.: «Sex and gender are different: Sexual identity and gender identity are different», Pacific Center for Sex and Society. Revision 5.1 (December 3, 2001) (en http://www.hawaii.edu/ PCSS/online_artcls/intersex/sexual_I_G_web.html).

66. DenNIS, C.: "The most important sexual organ», Nature, 427 (2004), pp. 390-392.

67. SWAAB, D.F.: «The Human Hypothalamus. Basic and Clinical Aspects. Part II: Neuropathology of the Hypothalamus and Adjacent Brain Structures», en M.J. Aminoff, F. Boller y D.F. Swaab (eds.): Handbook of Clinical Neurology, Amsterdam, Elsevier, 2004, p. 596.

68. BakKer, J.; De Mees, C.; Douhard, Q.; Balthazart, J.; Gaban, P.; Szpirer, J. y SzPirer, C.: "Alpha-fetoprotein protects the developing female mouse brain from masculinization and defeminization by estrogens", Nature Neuroscience, 9 (2006), pp. 220-226. 
Los niveles de testosterona en el suero de los embriones humanos comienza a detectarse entre la 12 y 18 semanas del embarazo ${ }^{69}$. Durante el desarrollo precoz de los niños se observan dos picos en los que los niveles séricos de testoterona están elevados. El primer pico tiene lugar hacia la mitad del embarazo de forma que entre las semanas 34 y 41 los niveles de testosterona de los chicos es 10 veces mayor que en las chicas ${ }^{70}$. El segundo pico de testosterona se produce en el tercer mes después del nacimiento. En efecto, al final del embarazo, cuando la $A F P$ cae, el feto está más expuesto a los estrógenos, producidos por la placenta, los cuales inhiben al eje-hipotálamo-hipofisario del niño. Esta inhibición se pierde después del nacimiento, lo que determina que las hormonas gonodatróficas induzcan la producción de un pico de testosterona en los niños $y$ un pico de estrógenos en las niñas ${ }^{71}$. El nivel de testosterona en niños, en este momento, es tan alto como en el adulto, aunque gran parte de ella circula unida a proteínas. En el caso de fetos femeninos y de niñas recién nacidas, no hay un nivel elevado de testosterona en ninguno de estos dos periodos.

Estos dos picos sirven para programar y organizar las estructuras y los circuitos del cerebro para el resto de la vida del sujeto. Al llegar la pubertad, las hormonas sexuales circulantes "activan» los circuitos y desencadenan la puesta en marcha de diferentes patrones de conducta, incluidos algunos trastornos latentes que se habían generado durante el desarrollo, como por ejemplo la esquizofrenia $^{72}$. Los cambios del cerebro, debidos a la interacción de las hormonas con el desarrollo neuronal, parecen ser la base de las diferencias de la identidad y el papel de género, de la orientación sexual, la cognición y las conductas agresivas, entre ambos sexos. Por tanto, aquellos factores que durante la vida intrauterina influyen en las interacciones entre las hormonas y el desarrollo del cerebro también pueden hacerlo, de manera permanente, sobre la conducta ulterior.

Son abundantes los datos que indican que las hormonas ejercen un efecto directo sobre el desarrollo del cerebro. Por ejemplo, en el síndrome de insensibilidad a los andrógenos (Androgen Insensitivity Sindrome o AIS), causado por una mutación del gen del receptor de estas hormonas, pacientes con un genotipo masculino (XY) se desarrollan fenotípicamente como mujeres que viven su orientación sexual y sus fantasías eróticas sin problemas de identidad de géne-

69. Finegan, J.A.; BARTLEMAN, B. y Wong, P.Y.: «A window for the study of prenatal sex hormone influences on postnatal development», The Journal of General Psychology, 150 (2006), pp. 267275.

70. De Zegher, F.; DeVlieger, H. y Veldhuis, J.D.: «Pulsatile and sexually dimorphic secretion of luteinizing hormone in the human infant on the day of birth", Pediatric research, 32 (1992), pp. 605-607.

71. QuigleY, C.A.: «The postnatal gonadotropin and sex steroid surge-insights from the androgen insensitivity síndrome», The Journal of Clinical Endocrinology and Metabolism, 87 (2002), pp. 24-28.

72. Colapinto J.: As Nature Made Him. The Boy Who was Raised as a Girl, New York, Harper Collins Publishers Inc, 2001. 
$\mathrm{ro}^{73}$. Otro ejemplo de la influencia hormonal sobre el desarrollo del cerebro sería el de fetos masculinos (XY) que con deficiencias de alguno de los enzimas del metabolismo de los esteroides sexuales, como la 5 alfa-reductasa-2 o la 17betahidroxiesteroide-dehidrogenasa-3, que les impide que la testosterona periférica se transforme en dihidrotestosterona, nacen con fenotipo de niñas pero dotadas con un clítoris muy grande. Estos recién nacidos varones (XY) son habitualmente reasignados como niñas. Sin embargo, cuando la testosterona aparece en la pubertad, de estos niños (XY) reasignados como niñas, su clítoris aumenta hasta el tamaño de un pene, los testículos descienden y se comienzan a masculinizar y a muscular. A pesar de que estos niños habían sido reasignados como niñas, la mayoría de ellos $(60 \%)$ cambian a varones heterosexuales cuando se hacen mayores $^{74}$, probablemente debido al efecto organizador de la testosterona en el desarrollo temprano de su cerebro. Un tercer ejemplo es el de aquellos niños (XY) que nacen con extrofia cloacal y que habitualmente también son resignados como niñas, al nacer. En dos recientes revisiones ${ }^{75}$ se muestra como cuando estos niños (reasignados como niñas) son adultos, solamente el $65 \%$ de ellos continuaron viviendo como niñas y que cuando de este grupo se excluyeron a los que sufrían disforia de género el porcentaje descendió hasta el $47 \%$. En resumen, estos ejemplos apuntan hacia la existencia de una acción directa de la testosterona en el desarrollo del cerebro de los niños y en el desarrollo de la identidad de género masculina y de la orientación sexual.

A pesar de que las hormonas sexuales son muy importantes para el desarrollo psicosexual y del SN, no son el único factor. Actualmente se sabe que actúan otros factores también, entre los que se postulan muchos genes. Concretamente, en el ratón se conocen 50 genes que se expresan a diferentes niveles del cere-

73. Wisnieswski, A.B.; Migeon, C.J.; Meyer,-BAhlburG, H.F.L.; GearharT, J.P.; BerkovitZ, G.D.; BROWN, T.R. y MONEY J.: "Complete androgen insensivity syndrome: long-term medical, surgical, and psychosexual outcome», The Journal of Clinical Endocrinology and metabolism, 85 (2000), pp. 2664-2669.

74. Wilson, J.D.; Griffin, J.E. y RusselL, D.W.: «Steroid 5a-reductase 2 deficiency», Endocrine Reviews, 14 (1993), pp. 577-593; Hughes, I.A.; Houk, C.; AHMED, S.F.; LeE, P.A.: "Consensus statement on management of intersex disorders", Archives of Diseases Childhood, 91 (2006), pp. 554-563; IMPERATO-MCGINLEY, J.; PETERSON, R.E. y GAUTIER, T.: «Male pseudohermaphroditism secondary to $5 \mathrm{a}$-reductase deficiency -a model for the role of androgens in both the development of the male phenotype and the evolution of a male gender identity", Journal of Steroid Biochemistry, 11(1979), pp. 637-645; COHEN-KeTTENIS, P.T.: «Gender change in 46,XY persons with 5a-reductase-2 deficiency and 17b-hydroxysteroid dehydrogenase-3 deficiency», Archives of Sexual Behavior, 34 (2005), pp. 399-410.

75. MeYer-BAHLBURG, H.F.L.: "Gender identity outcome in female-raised 46,XY persons with penile agenesis, cloacal exstrophy of the bladder, or penile ablation", Archives of Sexual Behavior, 34 (2005), pp. 423-438; REINER, W.G. y GEARHART, J.P.: «Discordant sexual identity in some genetic males with cloacal exstrophy assigned to female sex at birth", The New England Journal of Medicine, 350 (2004), pp. 333-341. 
bro de los machos y de las hembras ${ }^{76}$. También genes del cromosoma X pueden contribuir al desarrollo de la identidad de género y de la orientación sexual77.

Debido a que la diferenciación de los genitales tiene lugar en los dos primeros meses del embarazo y que la diferenciación sexual del cerebro comienza a partir del séptimo mes y se extiende hasta la edad adulta, es fácil deducir que ambos procesos pueden ser independientes. Este "décalage», entre la maduración genital y el desarrollo sexual del cerebro puede dar lugar a individuos con órganos sexuales masculinos e identidad de género femenina y viceversa, fenómeno que hemos descrito antes como transexualidad, y de cuyas bases neurobiológicas nos ocuparemos a continuación. Este desfase también explicaría por qué, en los casos de DSDs, el grado de masculinización de los genitales no siempre refleja el mismo grado de masculinización del cerebro ${ }^{78}$.

\subsection{Cerebro, identidad y papel de género y transexualidad}

Un aspecto clásico, en el que se observan claras diferencias entre niñas y niños, son las conductas relacionadas con el juego. En efecto, es un estereotipo, ampliamente extendido, que los niños son mucho más activos y rudos que las niñas y que prefieren juguetes distintos como coches y armas, por ejemplo. Por su parte, las niñas prefieren jugar con muñecas. Tradicionalmente, esta diferenciación de la conducta ha sido atribuida a la presión social. Sin embargo, cada vez hay más evidencias que reflejan la existencia de un factor endógeno, en este tipo de conductas. En efecto, diferencias similares también se dan en otros primates. Así, en un estudio realizado por Alexander y $\mathrm{Hines}^{79}$ se ofrecieron juguetes (muñecas, coches y balones) a un grupo de monos verdes (Cercopithecus aethiops). Las hembras del grupo elegían, de manera consistente, las muñecas y les examinaban sus genitales. Por su parte, los machos estaban más interesados en jugar con los cochecitos y con las pelotas. Sin embargo, tanto en los monos como en los humanos, la predilección por juguetes neutros (libros de pinturas, perros de juguete, etc.) no daban diferencias de elección entre ambos sexos.

En otros estudios ${ }^{80}$, chicas expuestas a elevadas dosis de andrógenos, durante el embarazo, debido a que padecían CAH, tendían a elegir, como compañe-

76. DeWING, P.; SHI, T.; HorVATH, S. y VILAIN, E.: «Sexually dimorphic gene expression in mouse brain precedes gonadal differentiation. Brain Research", Molecular Brain Research, 118 (2003), pp. $82-90$

77. LOPES, A.M.; Ross, N.; Close, J.; DAGnAll, A.; AMORIM, A. y GROW, T.J.: «Inactivation status of PCDH11X: sexual dimorphisms in gene expresión levels in brain», Human Genetics, 119 (2006), pp. 267-275.

78. SWAAB, D.F.: «The Human Hypothalamus. Basic and Clinical Aspects. Part II..., op. cit.

79. AleXANDER, G.M. y Hines, M.: "Sex differences in response to children's toys in nonhuman primates (Cercopithecus aethiops sabaeus)", Evolution and Human Behavior, 23 (2002), pp. 467479.

80. Nordenström, A.; Servin, A.; Bohlin, G.; Larsson, A. y Wedell, A.: «Sex-typed toy play behavior correlates with the degree of prenatal androgen exposure assessed by CYP21 genotype in girls with congenital adrenal hyperplasia», The Journal of Clinical Endocrinology and Metabolism, 87 (2002), pp. 5119-5124. 
ros de juegos, a niños y a jugar con los mismos juguetes que éstos, siendo su conducta más ruda que las de las otras niñas. Diferencias similares han sido encontradas cuando se analizan las preferencias por el tipo de dibujo a realizar ${ }^{81}$. Las chicas prefieren realizar figuras del cuerpo humano, fundamentalmente de su sexo, así como flores y mariposas. En sus dibujos utilizan colores brillantes (rojo, naranja y amarillo), organizan los objetos en hileras y, en general, realizan dibujos de aspecto pacífico. Por el contrario, los chicos prefieren dibujar objetos técnicos (coches, aviones, trenes, armas, etc.), como si estuvieran vistos desde el aire. En general, utilizan colores oscuros como el azul. Estos datos apuntan la idea de que las diferencias sexuales, en las conductas lúdicas, aparecen pronto en la evolución, antes de los homínidos, y parecen imprimirse durante la vida intrauterina, bajo la influencia de las hormonas sexuales.

Estudios realizadas con gemelos y familias sugieren la existencia de factores genéticos en el desarrollo de la transexualidad ${ }^{82}$. Entre los factores de carácter genético se han descrito algunas alteraciones cromosómicas raras. Igualmente se ha encontrado que polimorfismos de los genes para los receptores alfa y beta de los estrógenos y para la aromatasa pueden estar relacionados con un aumento de riesgo de transexualidad ${ }^{83}$.

Niveles anormales de hormonas sexuales, durante el desarrollo intrauterino temprano, también podrían jugar un papel en la génesis de la transexualidad como parece indicarlo la alta incidencia de ovarios poliquísticos, oligomenorrea y amenorrea en el grupo de transexuales FtM. Estas observaciones apuntan a que una temprana exposición del feto femenino (XX), a niveles anormalmente altos de testosterona ${ }^{84}$ pudieran haber sido el desencadenante de la situación transexual. Los siguientes ejemplos pueden servir para ilustrar esta afirmación:

(1) El riesgo que tiene una niña con síndrome de $\mathrm{CAH}$, expuesta a elevados niveles de testosterona en el útero materno, de ser transexual es hipotéticamente elevado. Sin embargo aunque en estos casos los niveles de testosterona son de 300 a 1000 veces mayores de lo normal, el riesgo real es sólo del 1 al 3\% ${ }^{85}$, y la probabilidad de padecer problemas serios de género del 5,2\% ${ }^{86}$. El consenso

81. LiJIMA, M.; ARISAKA, O. y MiNAMOTO, F.: «Sex differences, in children's free drawing: a study in girls with congenital adrenal hyperplasia», Hormones and Behavior, 40 (2001), pp. 90-104.

82. CoOlidge, F.L.; THede, L.L y YounG, S.E.: «The heritability of gender identity... op. cit. (Ver cita 54).

83. Henningsson, S.; WestberG, L.; NilsSON, S.; LundstrÖM, B.; EKSEliUs, L.; BOdLUnd, O.; LindSTROM, E.; Hellstrand, M.; ROSMOND, R.; ERIKKSON, E. y LANDÉN, M.: «Sex steroid-related genes and male-to-female transsexualism", Psychoneuroendocrinology, 30 (2005), pp. 657-664.

84. PADMANABHAn, V.; MANikKam, M.; ReCABARREn, S. y Foster, S.: "Prenatal testosterone excess programs reproductive and metabolic dysfunction in the female», Molecular and Cellular Endocrinology, 246 (2006), pp. 165-174.

85. Zucker, K.J.; Bradley, S.J.; Oliver, G.; Blake, J.; Fleming, S. y Hood, J.: «Psychosexual development of women with congenital adrenal hyperplasia", Hormones and Behavior, 30 (1996), pp. 300-318.

86. Dessens, A.B.; Cohen-Kettenis, P.T.; Mellenbergh, G.J.; Poll, N.; Koppe, J.G. y Boer, K.: «Prenatal exposure to anticonvulsants and psychosexual development», Archives of Sexual Behavior, 28 (1999), pp. 31-44. 
es, por lo tanto, que chicas con CAH deberán ser mantenidas como chicas aun cuando hayan sido masculinizadas por acción de la testosterona ${ }^{87}$.

(2) Un segundo ejemplo es el de las mujeres epilépticas que han sido tratadas con fenobarbital o difantoina, durante el embarazo, y en las que se ha observado que tienen aumentado el riesgo de tener hijos transexuales. En efecto, se sabe que ambas sustancias tienen la capacidad de cambiar el metabolismo de las hormonas sexuales y que pueden actuar en la diferenciación sexual del cerebro de los niños. En un grupo de 243 mujeres, expuestas a estas sustancias durante el embarazo ${ }^{88}$, se encontraron tres casos de niños transexuales y algunos otros niños más con problemas de género. Aunque, aparentemente, tres niños parece ser un número pequeño, sin embargo, estadísticamente representa una proporción elevada de casos para una condición tan rara. Por otra parte, también el dietiletilbestrol (DES) parece incrementar el número de casos de transexualidad ${ }^{89}$.

Frente a estas evidencias biomédicas sobre varios factores que parecen intervenir en la génesis de la transexualidad podemos decir, por el contrario, que no hay datos de que las influencias sociales postnatales puedan ser responsables de la transexualidad.

Todos estos datos han contribuido a generar la teoría de que la transexualidad se debe a la existencia de un «desfase» entre la diferenciación de los órganos sexuales, producida durante los primeros meses del embarazo, y la diferenciación del cerebro. Ambos procesos se presentan separados en el tiempo y es posible que utilicen diferentes mecanismos. De ser cierta esta teoría, se podría esperar que los transexuales FtM posean estructuras cerebrales con características masculinas y lo contrario los transexuales MtF. En una reciente revisión, $\mathrm{Swaab}^{90}$ reseña importantes cambios en la porción central de la stría terminalis (bed nucleus of the stria terminalis, BSTc), una estructura que en las ratas está implicada en muchos aspectos de la conducta sexual. En humanos esta diferencia también es muy clara. En los hombres, la BSTc es el doble de grande que en las mujeres y contiene el doble de neuronas con somatostatina, aunque no se han encontrado diferencias en el número de neuronas de esta área, en relación con la orientación sexual. En los transexuales MtF, el BSTc aparece con características completamente femeninas. Tan sólo se conoce un caso de transexual FtM en el que se haya estudiado este núcleo y en él se observan características masculinas ${ }^{91}$. Parece bastante claro que las diferencias sexuales del BSTc no se deben a cambios en los niveles de hormonas en el adulto ${ }^{92}$.

87. HUGHES, I.A., et al.: «Consensus statement on management ..., op. cit.

88. DESSENS, A.B., et al.: «Prenatal exposure to anticonvulsants ..., op. cit.

89. KERLIN, S.: DES Action USA; DES Sons' information page, http://www.desaction.org/sons.htm; 2005.

90. SWAAB, D.C.: «Sexual diferentation of thebrain and behavior», Best Practice \& Research Clinical Endocrinology \& Metabolism, 21 (2007), pp. 431-444.

91. Ibíd., pp. 431-444.

92. ZHOU, J.N.; HOFMAN, M.A.; GOOREN, L.J.G. y SwAAB, D.F.: «A sex difference in the human brain and its relation to transsexuality", Nature, 378 (1995), pp. 68-70; KRUIJVER, F.P.M.; ZHOU, J.N.; Pool, C.W.; Hofman, M.A.; GOOREn, L.J. y SWAAB, D.F.: «Male-to-female transsexuals have 
Evidentemente, existen otras estructuras cerebrales que presentan dimorfismo sexual, así como importantes diferencias relacionadas con la identidad y el papel de género. Sin embargo, no es un objetivo de este articulo revisar exhaustivamente el tema, sino reflexionar sobre la importancia de los aspectos biológicos sobre sexo, género y conducta.

\subsection{Factores neurobiológicos de la orientación sexual}

También la orientación sexual parece estar determinada por factores que actúan durante el desarrollo intrauterino, en la interacción entre las hormonas sexuales y el cerebro. También aquí, los factores genéticos han sido analizados en gemelos y en familias. El componente genético de la homosexualidad, en ambos sexos, representa un $50 \%{ }^{93}$. Ahora bien, todavía es un problema por resolver qué genes intervienen en ella. A pesar de que los homosexuales tienen una tasa de procreación más baja que los otros miembros de la población, resulta llamativo ver cómo el factor genético de la homosexualidad se ha perpetuado a lo largo de la evolución. Una explicación de este fenómeno podría ser que este factor tenga, a su vez, efectos beneficiosos sobre la fecundidad del grupo. Según los estudios de Camperio-Ciani et al..$^{94}$, el factor genético de la homosexualidad masculina se transmite a través de la madre y proporciona, a su vez, una mayor fecundidad en las mujeres.

También los niveles anormalmente elevados de hormonas sexuales, durante la vida intrauterina, pueden influir sobre la orientación sexual, como se deduce del alto porcentaje de chicas bisexuales y homosexuales entre las que padecen $\mathrm{CAH}^{95}$. Otro ejemplo de la influencia de las hormonas sexuales sobre la orientación sexual son los estudios derivados del uso del dietiletilbestrol (DES). En efecto, entre 1939 y 1960, unos dos millones de mujeres norteamericanas y europeas consumieron DES como tratamiento para prevenir abortos. El DES es una sustancia análoga a los estrógenos que, en pequeñas dosis, no sólo produce un elevado riesgo de padecer cáncer de cuello uterino, sino de incrementar la incidencia de bisexualidad y homosexualidad, en niñas nacidas de madres que habían consumido $\mathrm{DES}^{96}$.

female neuron numbers in a limbic nucleus», The Journal of Clinical Endocrinology and Metabolism, 85 (2000), pp. 2034-2041. SWAAB, D.C.: «Sexual diferentation of the brain..., op. cit.

93. LEVAY, S. y HAMER, D.H.: "Evidence for a biological influence in male homosexuality», Scientific American, 270 (1994), pp. 44-49.

94. CAMPerio-Ciani, A.; Corna, F. y CAPILUPPI, C.: «Evidence for maternally inherited factors favouring male homosexuality and promoting female fecundity", Proceedings, Biological Sciences, 271 (2004), pp. 2217-2221.

95. ZUCKer, KJ.; BrADley, S.J.; OlIVER, G. et al.: «Psychosexual development of women..., op. cit.; MeYer-BAHLbURG, H.F.L.; Gruen. R.S. y NeW, M.I.: "Gender change from female to male in classical congenital adrenal hyperplasia», Hormones and Behavior, 30 (1996), pp. 319-332.

96. MeYer-BAHLBURG, H.F.L.; EHRHARDT, A.A. y ROSEN, L.R.: «Prenatal estrogens and the development of homosexualmorientation", Developmental Psychology, 31 (1995), pp. 12-21; EHRHARDT, A.A.; MEYer-BAHLBURG, H.F.L. y ROSEN, L.R.: «Sexual orientation after prenatal exposure to exogenous estrogen", Archives of Sexual Behavior, 14 (1985), pp. 57-75; TITUS-ERnSTOFF, L.; Perez, K.; Hatch, E.E.; Troisi, R.; Palmer, J.R.; Hartge, P.; Hyer, M.; Kaufman, R.; Adam, 
También la exposición prenatal a diferentes sustancias tóxicas, como nicotina, anfetaminas $\mathrm{u}$ hormonas tiroideas, incrementan el riesgo de tener hijas lesbianas ${ }^{97}$. Otro dato de interés es el hecho de que el estrés, durante el embarazo, aumenta el riesgo de tener un hijo varón homosexual ${ }^{98} \mathrm{o}$ una hija lesbiana ${ }^{99}$. No hay, sin embargo, evidencias sólidas respecto a su intervención durante el desarrollo postnatal. Por el contrario, niños que han nacido, por inseminación artificial, a demanda de una pareja de lesbianas, se desarrollan con una orientación heterosexual ${ }^{100}$. No hay evidencias sobre la idea de que la homosexualidad masculina es el producto del aprendizaje social, como por ejemplo el derivado de una educación femenina o la idea de que se trata de un estilo de vida elegido ${ }^{101}$.

Otro factor importante en la determinación de la orientación sexual es el puesto que se ocupa entre los hermanos. La probabilidad de que un muchacho pueda ser homosexual se incrementa con el número de hermanos mayores que tenga. Este fenómeno se explicaría por la respuesta inmunológica de la madre contra un producto del cromosoma Y de los hijos. La posibilidad de esta respuesta contra los factores masculinos se incrementa con cada embarazo de un varón ${ }^{102}$.

Hay muchos datos clínicos que indican cómo un buen número de las estructuras cerebrales están implicadas en la orientación sexual. En efecto, pacientes con el síndrome de Klüver-Bucy, cuyo lóbulo temporal está afectado, cambian su orientación de heterosexual a homosexual. Tumores del lóbulo temporal y del hipotálamo han sido relacionados con cambios de la orientación sexual (a homosexualidad o a pedofilia). En animales de experimentación, como el hurón y la rata, lesiones en el área preóptica del hipotálamo producen cambios en la orientación sexual ${ }^{103}$.

E.; Strohsnitter, W.; Noller, K.; PicketT, K.E. y Hoover, R.: «Psychosexual characteristics of men and women exposed prenatally to diethylstilbestrol», Epidemiology, 14 (2003), pp. 155160.

97. ElLis, L. y COle-HARDing, S.: «The effects of prenatal stress, and of prenatal alcohol and nicotine exposure, on human sexual orientation", Physiology and Behavior, 74 (2001), pp. 213-226; Ellis, L. y HellberG, J.: "Fetal exposure to prescription drugs and adult sexual orientation", Personality and Individual Differences, 38 (2005), pp. 225-236.

98. Ellis, L. y COlE-HARDING, S.: "The effects of prenatal stress ..., op. cit.; ElLIS, L.; AMES, M.A.; PECKHAM, W. y BURKE, D.: "Sexual orientation of human offspring may be altered by severe maternal stress during pregnancy", Journal of Sex Research, 25 (1988), pp. 152-157.

99. BAILEY, J.M.; WiLleRMAN, L. y PARKS, C.: "A test of the maternal stress theory of human male homosexuality", Archives of Sexual Behavior, 20 (1991), pp. 277-293.

100. GReEN, R.: "Sexual identity of 37 children raised by homosexual or transsexual parents», The American Journal of Psychiatry, 135 (1978), pp. 692-697.

101. LEVAY, S.: Queer Science. The Use and Abuse of Research into Homosexuality, Cambridge, MA, USA, The MIT Press, 1996.

102. BLANCHARD, R.: «Fraternal birth order and the maternal immune hypothesis of male homosexuality", Hormones and Behavior, 40 (2001), pp. 105-114. BOGAERT, A.F.: "The interaction of fraternal birth order and body size in male sexual orientation", Behavioral Neuroscience, 117 (2003), pp. 381-384.

103. SWAAB, D.F.: "The Human Hypothalamus. Basic and Clinical Aspects. Part I: Nuclei of the Hypothalamus", en M.J. Aminoff, F. Boller, y D.F. Swaab (eds.): Handbook of Clinical Neurology, Amsterdam, Elsevier, 2003, p. 476; SwAAB, D.F.: «The Human Hypothalamus. Basic and Clinical Aspects. Part II..., op. cit. 
El dimorfismo estructural y funcional del cerebro, relacionado con la orientación sexual, está ampliamente extendido. Una de las localizaciones donde se observan claras diferencia es en el núcleo supraquiasmático (SCN), considerado el reloj del SNC. Este núcleo presenta el doble de tamaño en los hombres homosexuales que en los hombres heterosexuales ${ }^{104}$. En experimentos con ratas se puede observar una diferencia similar inducida experimentalmente. El experimento produce ratas bisexuales adultas que tienen un mayor número de neuronas en su $\mathrm{SCN}^{105}$. En 1991, LeVay ${ }^{106}$ describió que el área INHA-3 del hipotálamo de los varones homosexuales y de las mujeres heterosexuales es menor que la de los varones heterosexuales. Por el contrario, la comisura anterior de los varones homosexuales y de las mujeres, implicadas en actividades cognitivas y del lenguaje, es mayor que la de los varones heterosexuales ${ }^{107}$.

Técnicas de Resonancia Magnética Funcional (fMRIs) han puesto de manifiesto diferencias del hipotálamo en relación con la orientación sexual. En hombres homosexuales, la actividad del hipotálamo no responde a los antidepresivos clásicos, (fluoxetina), como lo hace el hipotálamo de los hombres heterosexuales, los cuales manifiestan un tipo diferente de actividad en su sistema serotoninégico ${ }^{108}$. Otros estudios analizan las respuestas hipotalamicas frente a feromonas en relación con la orientación sexual ${ }^{109}$.

\section{CONCLUSIONES}

Tras el análisis que acabamos de realizar cabrían muchas conclusiones y controversias. Sin embargo, fieles a los planteamientos de la introducción, nuestra intención primordial ha sido introducir la idea de que los humanos también somos organismos y que en el abordaje de cualquiera de los temas que nos conciernen, como los aquí tratados, no debemos olvidar nuestra faceta biológica, mirada sin aprensión y sin la distorsión de componentes ideológicos, ajenos a la razón y al espíritu científico. Por otra parte, desde el punto de vista de la ciencia, tampoco cabe sacralizar las evidencias científicas y utilizarlas como mera munición de confrontación frente a opiniones y creencias distintas. En fin, se trata

104. SWAAB, D.F. y HOFMAN, M.A.: "An enlarged suprachiasmatic nucleus in homosexual men", Brain Research, 537 (1990), pp. 141-148.

105. SwaAb, D.F.; Slob, A.K.; Houtsmuller, E.J.; BrandT, T. y ZHOU, J.N.: «Increased number of vasopressin neurons in the suprachiasmatic nucleus ( $\mathrm{SCN}$ ) of 'bisexual' adult male rats following perinatal treatment with the aromatase blocker ATD", Brain Research. Developmental Brain Research, 85 (1995), pp. 273-279.

106. LEVAY, S.: «A difference in hypothalamic structure between heterosexual and homosexual men", Science, 253 (1991), pp. 1034-1037.

107. AlLEN, L.S. y GORSKI, R.A.: "Sexual orientation and the size of the anterior commissure in the human brain", PNAS, 89 (1992), pp. 7199-7202.

108. Kinnunen, L.H.; MOLTZ, H.; METZ, J. y COOPER, M.: "Differential brain activation in exclusively homosexual and heterosexual men produced by the selective serotonin reuptake inhibitor, fluoxetine», Brain Research, 1024 (2004), pp. 251-254.

109. BeRGLUND, H.; LindSTRÖM, P. y SAVIC, I.: "Brain response to putative pheromones in lesbian women", PNAS, 103 (2006), pp. 8269-8274. 
Sexo, género y biología

de establecer el dialogo entre las Dos culturas de las que hablaba Snow ${ }^{110}$, para converger en una Tercera cultura ${ }^{111}$, más interdisciplinar y humana.

110. Snow, C.P.: The two cultures, Cambridge, Cambridge University Press, 1998.

111. BrockmAn, J.: La tercera cultura, Barcelona, Tusquets Editores, 1995. 